\title{
EFECTOS DEL LASER Y LOS RAYOS X EN METALES
}

\author{
Oscar S. Monroy C., Mateo Márquez J., Régulo Sabrera A., y Arnaldo Huaroto S. \\ ${ }^{a}$ Departamento de Física Nuclear, Atómica y Molecular. Facultad de Ciencias Físicas, \\ Universidad Nacional Mayor de San Marcos. Apartado postal 14-0149, Lima 14 - Perú.
}

\begin{abstract}
Resumen
La interacción de sólidos con dos radiaciones (láser y rayos X) simultáneas es analizada utilizando técnicas teóricas no convencionales. Se calcula la razón de transición entre bandas de energía inducida por un láser con polarización circular y de intensidad arbitraria y por rayos-X. Para intensidades de láser moderadamente altas el comportamiento de la razón de transición obtenido utilizando el método convencional de perturbaciones es comparable al obtenido utilizando la aproximación de Volkov.
\end{abstract}

Palabras claves: Transición interbanda, metales, rayos X, láser, Volkov.

\begin{abstract}
Interaction between solids and two radiations (X-rays and laser) simultaneously is analyzed using non conventional theoretical techniques. In this way, it is calculated induced transition ratio in interbands by X-rays and circular polarized laser with arbitrary intensity. In the case of high moderated intensity laser, it was utilized conventional method of perturbations, so it produced a transition ratio similar to the obtained for Volkov approximation.
\end{abstract}

Keywords: Interband transition, metals, X-rays, laser, Volkov.

\section{Introducción.}

Un electrón en un sólido, en la representación de la masa efectiva, tiene propiedades que se asemejan a un electrón libre. Esto es razonable para electrones de la banda de conducción los cuales no están fuertemente ligados a los átomos del cristal. Por tanto, podemos analizar la excitación de un electrón de valencia dentro de la banda de conducción bajo la acción de dos campos de radiación: láser con polarización circular y de rayos-X.

Se asume que el electrón de valencia puede ser representado por una función de onda tipo Volkov [1]. También se asume que las superficies de energía de las bandas de conducción tienen simetría esférica. Además, se utiliza la aproximación dipolar eléctrica para los campos de radiación [2].

En la sección 2 se utiliza el formalismo de la matriz de transición para calcular la razón de transición para la excitación (por $\mathrm{n}$ fotones de láser) de un electrón de valencia dentro de la banda de conducción. Se obtienen resultados analíticos de forma cerrada para valores arbitrarios de la intensidad del láser. En la sección 3 se calcula la razón de transición utilizando la teoría de perturbaciones y en la sección 4 se comparan los resultados obtenidos por los dos métodos de cálculo descritos. 


\section{Razón de transición en la aproximación de Volkov}

Puesto que el electrón de conducción tiene más carácter de electrón libre que de electrón de valencia, el elemento de matriz que describe una transición entre los estados inicial (i) y final (f) en la presencia de un campo electromagnético está dado (en unidades naturales $\hbar=\mathrm{c}=1$ ) por

$$
S_{f i}=-i \int_{-\infty}^{+\infty}\left\langle\Psi_{f}(t)\left|H^{\prime}(t)\right| \Psi_{i}(t)\right\rangle d t
$$

siendo $\left|\Psi_{\mathrm{f}}(\mathrm{t})\right\rangle$ el estado final dependiente del campo externo para el electrón de conducción, y $\left|\Psi_{\mathrm{i}}(\mathrm{t})\right\rangle$ es el estado inicial libre del campo externo para el electrón de valencia, y

$$
\begin{aligned}
& H^{\prime}(t)=-e \vec{r} \cdot \vec{E}_{x}(\overrightarrow{0}, t) \\
& H^{\prime}(t)=-e \vec{r} \cdot \vec{E}_{o x} \operatorname{sen} \omega_{x} t
\end{aligned}
$$

es el hamiltoniano de interacción dipolar eléctrica independiente del "gauge" (calibración) [2].

El estado inicial para el electrón de valencia es simplemente la función de Bloch estándar

$$
\Psi_{i}(\vec{r}, t)=\frac{1}{\sqrt{N}} u_{v, \vec{k}} \cdot(\vec{r}) e^{i\left(\vec{k} \cdot \cdot \vec{r}-E_{v} t\right)}
$$

siendo $\mathrm{N}$ el número de celdas unitarias en el cristal, $\overrightarrow{\mathrm{k}}$ ' el vector de onda y

$$
\mathrm{E}_{v}=-\mathrm{E}_{\mathrm{g}}-\frac{\mathrm{k}^{{ }^{2}}}{2 \mathrm{~m}_{v}}
$$

la energía en la aproximación de la masa efectiva con banda de simetría esférica. Las

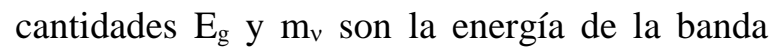
prohibida y la masa efectiva, respectivamente.

Para el estado final se utiliza una función tipo Volkov para representar al electrón de conducción de modo que involucre a la luz láser con polarización circular, y está dado por

$$
\begin{aligned}
& \Psi_{\mathrm{f}}(\overrightarrow{\mathrm{r}}, \mathrm{t})=\frac{1}{\sqrt{\mathrm{N}}} \mathrm{u}_{\mathrm{c}, \overrightarrow{\mathrm{k}}}(\overrightarrow{\mathrm{r}}) \\
& \times \exp \left\{\mathrm{i}\left[\overrightarrow{\mathrm{k}} \cdot \overrightarrow{\mathrm{r}}+\xi \operatorname{sen}\left(\omega_{\mathrm{L}} \mathrm{t}-\rho\right)-\mathrm{E}_{\mathrm{c}} \mathrm{t}\right]\right\}
\end{aligned}
$$

donde,

$$
\begin{aligned}
& E_{c}=\frac{k^{2}}{2 m_{c}}+\frac{\left(\mathrm{e} \mathrm{A}_{O L}\right)^{2}}{4 \mathrm{~m}_{\mathrm{c}}} \\
& \zeta=\frac{\mathrm{e} \mathrm{A}_{0 \mathrm{~L}}\left(\mathrm{k}_{\mathrm{x}}^{2}+\mathrm{k}_{\mathrm{y}}^{2}\right)}{\sqrt{2} \omega_{\mathrm{L}} \mathrm{m}_{\mathrm{c}}} \\
& \rho=\tan ^{-1}\left(\mathrm{k}_{\mathrm{y}} / \mathrm{k}_{\mathrm{x}}\right)
\end{aligned}
$$

Utilizando una expansión de la función de Bessel para el término exp[i $\left.\xi \operatorname{sen}\left(\omega_{\mathrm{L}} \mathrm{t}-\rho\right)\right]$, la integración temporal para el elemento de matriz da

$$
\begin{aligned}
& \mathrm{S}_{\mathrm{fi}}=-\mathrm{i} \pi \mathrm{e} \mathrm{E}_{\mathrm{ox}} \delta_{\overrightarrow{\mathrm{k}}, \overline{\mathrm{k}}} \cdot \sum_{\ell=-\infty}^{\infty}(-1)^{\ell} \mathrm{J}_{\ell}(\zeta) \mathrm{e}^{-\mathrm{i} \ell \rho} \\
& \times\left\{\mathrm{M}_{-} \delta\left[\mathrm{E}_{\mathrm{c}}-\mathrm{E}_{\mathrm{v}}+(\ell+1) \omega_{\mathrm{L}}+\omega_{\mathrm{x}}\right]+\right. \\
& \mathrm{M}_{+} \delta\left[\mathrm{E}_{\mathrm{c}}-\mathrm{E}_{\mathrm{v}}+(\ell-1) \omega_{\mathrm{L}}+\omega_{\mathrm{x}}\right\}
\end{aligned}
$$

siendo,

$$
M_{ \pm}=\left\langle u_{c}(\vec{r})|x \pm i y| u_{v}(\vec{r})\right\rangle
$$

y la integración para los elementos de matriz M es sobre una celda unitaria simple del cristal.

La matriz en la Ec.(9) puede utilizarse para describir la absorción de $\mathrm{n}$ fotones del láser. Por tanto,

$$
\ell \pm 1=-\mathrm{n} .
$$

Con esta restricción la matriz $\mathrm{T}$ puede ser escrita ( en unidades cgs ) así :

$$
\begin{aligned}
& \mathrm{T}_{\mathrm{fi}}^{(\mathrm{n})}=\left(\frac{\mathrm{eE} \text { ox }}{2^{3 / 2} \mathrm{mc}}\right) \delta_{\bar{k}, \bar{k},} \cdot e^{\mathrm{in} \rho} \\
& \times\left[\mathrm{J}_{\mathrm{n}+1}(\zeta) \exp (\mathrm{i} \rho) \mathrm{M}_{-}+\mathrm{J}_{\mathrm{n}-1}(\zeta) \exp (-\mathrm{i} \rho) \mathrm{M}_{+}\right]
\end{aligned}
$$


Utilizando la relación dada por: $\delta_{\overrightarrow{\mathrm{k}}, \overrightarrow{\mathrm{k}}}^{2}=\delta_{\overrightarrow{\mathrm{k}}, \overrightarrow{\mathrm{k}}}$; $\left|\mathrm{T}_{\mathrm{fi}}^{(\mathrm{n})}\right|^{2}$ queda en la forma

$$
\begin{aligned}
& \left|\mathrm{T}_{\mathrm{fi}}^{(\mathrm{n})}\right|^{2}=\left(\frac{\mathrm{eE} o \mathrm{ox}}{\mathrm{mc}}\right)^{2} \delta_{\vec{k}, \vec{k}} \cdot \frac{1}{8}\left\{\left|\mathrm{M}_{-}\right|^{2} \mathbf{J}_{\mathrm{n}+1}^{2}(\zeta)+\right. \\
& \left|\mathbf{M}_{+}\right|^{2} \mathbf{J}_{\mathrm{n}-1}^{2}(\zeta)+2 \mathrm{~J}_{\mathrm{n}+1}(\zeta) \mathbf{J}_{\mathrm{n}-1}(\zeta) \\
& \left.\times\left[\left(\mathrm{M}_{\mathrm{x}}^{2}-\mathrm{M}_{\mathrm{y}}^{2}\right) \cos 2 \rho+2 \mathrm{M}_{\mathrm{x}} \mathrm{M}_{\mathrm{y}} \operatorname{sen} 2 \rho\right]\right\}
\end{aligned}
$$

donde los elementos de matriz $\mathrm{M}_{\mathrm{x}}, \mathrm{M}_{\mathrm{y}}$ se deducen de la Ec.(10).

La razón de transición está dado por

$$
\begin{gathered}
\omega_{\mathrm{fi}}^{(\mathrm{n})}=\frac{2 \pi}{\hbar} \sum_{\mathrm{k}, \mathrm{k}^{\prime}}\left|\mathrm{T}_{\mathrm{fi}}^{(\mathrm{n})}\right|^{2} \delta\left(\mathrm{E}_{\mathrm{f}}-\mathrm{E}_{\mathrm{i}}-\mathrm{n} \hbar \omega_{\mathrm{L}}\right. \\
\left.-\hbar \omega_{\mathrm{x}}\right)
\end{gathered}
$$

De las ecs.(13) y (14) la razón de transición por unidad de volumen es

$$
\begin{aligned}
& \mathrm{W}^{(\mathrm{n})}=\frac{\left(\mathrm{eE} \mathrm{ox}_{\mathrm{x}} / \mathrm{mc}\right)^{2}}{8(2 \pi)^{2} \hbar} \int_{\mathrm{ZB}}\left\{\left|\mathrm{M}_{-}\right|^{2} \mathrm{~J}_{\mathrm{n}+1}^{2}(\zeta)+\right. \\
& +\left|\mathrm{M}_{+}\right|^{2} \mathrm{~J}_{\mathrm{n}-1}^{2}(\zeta)+2 \mathrm{~J}_{\mathrm{n}+1}(\zeta) \times\left[\left(\mathrm{M}_{\mathrm{x}}^{2}-\right.\right. \\
& \left.\left.\left.\mathrm{M}_{\mathrm{y}}^{2}\right) \cos 2 \rho+2 \mathrm{M}_{\mathrm{x}} \mathrm{M}_{\mathrm{y}} \operatorname{sen} 2 \rho\right]\right\} \\
& \times \delta\left(\mathrm{E}_{\mathrm{c}}-\mathrm{E}_{\mathrm{v}}-\mathrm{n} \hbar \omega_{\mathrm{L}}-\hbar \omega_{\mathrm{x}}\right) \mathrm{d}^{3} \mathrm{k}
\end{aligned}
$$

donde la integración sobre la zona de Brillouin (ZB) representa una suma sobre los estados finales [3].

Asumiendo que los elementos de matriz M son independientes de $\overrightarrow{\mathrm{k}}$, la razón de transición por unidad de volumen resulta

$$
\begin{aligned}
& \mathrm{W}^{(\mathrm{n})}=\frac{\mathrm{B}(\lambda / \sqrt{2})^{2 \mathrm{n}}}{\mathrm{y}_{\mathrm{o}}(\mathrm{n}-1) !(2 \mathrm{n}-1) !}\left\{\left|\mathrm{M}_{+}\right|^{2}\right. \\
& \times \mathrm{F}_{2}\left(\mathrm{n}-\frac{1}{2} ; 2 \mathrm{n}-1, \mathrm{n}+\frac{1}{2} ;-\lambda^{2}\right) \\
& +\frac{(\lambda / \sqrt{2})^{4}\left|\mathrm{M}_{-}\right|^{2}}{\mathrm{n}(\mathrm{n}+1)(2 \mathrm{n}+1)(2 \mathrm{n}+3)} \\
& \left.\times \mathrm{F}_{2}\left(\mathrm{n}+\frac{3}{2} ; 2 \mathrm{n}+3, \mathrm{n}+\frac{5}{2} ;-\lambda^{2}\right)\right\}
\end{aligned}
$$

siendo $\mathrm{F}_{2}(\mathrm{a} ; \mathrm{b} ; \mathrm{c} ; \mathrm{z})$ la función hipergeométrica, y además

$$
\begin{aligned}
& B=\frac{\left(m_{c} \omega_{L}\right)^{2} m_{V}}{2 \pi \hbar^{3} m^{2}} \\
& y=\frac{1}{\hbar}\left[2 m_{V}\left(n \hbar \omega_{L}+\hbar \omega_{x}-E_{g}-\frac{\left(e A_{0 L} / c\right)^{2}}{4 m_{c}}\right]^{1 / 2}\right. \\
& \lambda=e A_{0 L} y / \sqrt{2} \omega_{L} c m_{c}
\end{aligned}
$$

\section{Razón de transición según la teoría de perturbaciones.}

El elemento de matriz de orden j el cual describe una transición entre los estados inicial (i) y final (f) viene dado (en unidades naturales $(\hbar=\mathrm{c}=1$ ) por:

$\mathrm{S}_{\mathrm{fi}}^{(\mathrm{j})}=-\mathrm{i} \int_{-\infty}^{+\infty}\left\langle\varphi_{\mathrm{f}}(\mathrm{t})|\mathrm{H} *(\mathrm{t})| \psi_{\mathrm{i}}^{\mathrm{j}}(\mathrm{t})\right\rangle \mathrm{dt}$

También, usaremos la perturbación tradicional, dependiente del "gauge" (calibración), en la aproximación dipolar:

$$
\mathrm{H} *(\mathrm{t})=-\frac{\mathrm{e}}{\mathrm{m}} \overrightarrow{\mathrm{A}}_{\mathrm{L}}(\overrightarrow{0}, \mathrm{t}) \cdot \overrightarrow{\mathrm{p}}
$$

Para la absorción de un láser polarizado circularmente la parte de $\mathrm{H}^{*}(\mathrm{t})$ que contribuye es:

$$
\mathrm{H} *(\mathrm{t})=\mathrm{H}_{1} \mathrm{e}^{-\mathrm{i} \omega_{\mathrm{L}} \mathrm{t}}
$$


donde

$$
\mathrm{H}_{1}=\frac{\mathrm{e} \mathrm{A}_{0 \mathrm{~L}}}{2^{3 / 2} \mathrm{~m}}\left(\mathrm{p}_{\mathrm{x}}+\mathrm{i} \mathrm{p}_{\mathrm{y}}\right)
$$

Bajo estas restricciones el estado inicial de orden $\mathrm{j}$ es:

$$
\begin{aligned}
& \psi_{i}^{(j)}(t)=\varphi_{i}(t)+\sum_{r_{1}, \ldots, r_{i}}\left\{\varphi_{r_{j}}(t)\right. \\
& \times \frac{r_{j}\left|H_{1}\right| r_{j-1}\langle\ldots\rangle r_{1}\left|H_{1}\right| i\langle}{\left(E_{r_{j}}-E_{i}-j \omega_{L}\right) \ldots\left(E_{n_{1}}-E_{i}-\omega_{L}\right)} \\
& \left.\times \exp \left[i\left(E_{r_{j}}-E_{i}-j \omega_{L}\right) t\right]\right\}
\end{aligned}
$$

Aquí los estados $\left|\mathrm{r}_{\mathrm{j}}\right\rangle$ corresponden a la banda de conducción con valores de $\overrightarrow{\mathrm{k}}$ diferentes. Las funciones de onda $\varphi(t)$ están dadas por las funciones de Bloch en la Ec.(3).

La matriz $\mathrm{T}$ para la absorción de $\mathrm{n}$ fotones de láser se obtiene de las ecs.(18) y (22) en la forma:

$$
\mathrm{T}_{\mathrm{fi}}^{(\mathrm{n})}=\sum_{\mathrm{r}_{1}, \ldots, \mathrm{r}_{\mathrm{n}-1}} \frac{\left\langle\mathrm{f}\left|\mathrm{H}_{1}\right| \mathrm{r}_{\mathrm{N}-1}\right\rangle \ldots\left\langle\mathrm{r}_{1}\left|\mathrm{H}_{1}\right| \mathrm{i}\right\rangle}{\left[\mathrm{E}_{\mathrm{n}-1}-\mathrm{E}_{\mathrm{i}}-(\mathrm{n}-1) \omega_{\mathrm{L}}\right]}
$$

Debido a la conservación del momentum, la suma sobre los estados intermedios, en la Ec.(23), puede realizarse explícitamente para obtener (en unidades cgs) :

$$
\begin{aligned}
& \mathrm{T}_{\mathrm{fi}}^{(\mathrm{n})}=\left(\frac{\mathrm{e} \mathrm{A}_{\mathrm{ox}}}{2^{3 / 2} \mathrm{c}}\right)^{\mathrm{n}}\left(\frac{\hbar}{\mathrm{m}_{\mathrm{c}}}\right)^{\mathrm{n}-1}\left(\frac{\mathrm{M}_{+}^{*}}{\mathrm{~m}}\right) \mathrm{k}_{+}^{\mathrm{n}-1} \delta_{\overrightarrow{\mathrm{k}}_{i}, \mathrm{k}_{\mathrm{f}}} \\
& {\left[\pi_{\mathrm{j}=1}^{\mathrm{n}-1}\left(\frac{\hbar^{2} \mathrm{k}_{\mathrm{f}}^{2}}{2 \mathrm{~m}_{\mathrm{r}}}+\mathrm{E}_{\mathrm{g}}-\mathrm{j} \hbar \omega_{\mathrm{L}}\right)\right]^{-1}}
\end{aligned}
$$

donde

$$
\begin{aligned}
& \mathrm{M}_{+}^{*}=\left\langle\mathrm{u}_{\mathrm{c}}(\overrightarrow{\mathrm{r}})\left|\left(\mathrm{p}_{\mathrm{x}}-\mathrm{ip} \mathrm{p}_{\mathrm{y}}\right)\right| \mathrm{u}_{\mathrm{v}}(\overrightarrow{\mathrm{r}})\right\rangle \\
& \mathrm{k}_{+}=\mathrm{k}_{\mathrm{x}}+\mathrm{ik} \mathrm{k}_{\mathrm{y}}
\end{aligned}
$$

Utilizando las Ecs.(14) y (24), la razón de transición puede ser escrita así:

$$
\begin{aligned}
& \mathrm{W}_{\mathrm{o}}^{(\mathrm{n})}=(2 \pi)^{-2} \hbar^{-1}\left(\frac{\mathrm{e} \mathrm{A}_{0 \mathrm{~L}}}{2^{3 / 2} \mathrm{c}}\right)\left(\frac{\hbar}{\mathrm{m}_{\mathrm{c}}}\right)^{2(\mathrm{n}-1)} \\
& \left(\frac{\left|M_{+}\right|}{m}\right) \iint_{Z B}\left|K_{+}\right|^{2(n-1)} \int\left(\frac{\hbar^{2} k^{2}}{m_{r}}+E_{g}-\right. \\
& \left.n \hbar \omega_{L}-\hbar \omega_{x}\right)\left[\pi _ { j = 1 } ^ { n - 1 } \left(\frac{\hbar^{2} k^{2}}{2 m_{p}}+E_{g}-\right.\right. \\
& \left.\left.\mathrm{j} \hbar \omega_{\mathrm{L}}-\hbar \omega_{\mathrm{x}}\right)\right]^{-2} \mathrm{~d}^{3} \mathrm{k}
\end{aligned}
$$

Utilizando la conservación del momentun y despreciando la dependencia de $\overrightarrow{\mathrm{k}}$ en $\mathrm{M}_{+}^{*}$, la integral en la Ec.(26) puede realizarse utilizando coordenadas cilíndricas; el resultado de las operaciones es :

$$
\begin{aligned}
& W_{o}^{(n)}=\frac{(2 \pi)^{n-1}\left(\left|M_{+}^{*} / m\right|^{2}\left(e^{2} / c\right)^{n}\right.}{2(n-1) !(2 n-1) ! n_{o}^{n} \hbar^{2(n+1)}} \\
& \times \frac{\left(2 m_{r}\right)^{(2 n+1) / 2}\left(n \hbar \omega_{L}+\hbar \omega_{x}-E_{g}\right)^{(2 n-1) / 2} I^{n}}{\omega_{L}^{2(2 n-1)} m_{c}^{2(n-1)}}
\end{aligned}
$$

donde $\mathrm{I}=\mathrm{n}_{\mathrm{o}} \omega_{\mathrm{L}}^{2} \mathrm{~A}_{0 \mathrm{~L}}^{2} / 8 \pi \mathrm{c}$ es la intensidad del láser $\mathrm{y}_{\mathrm{o}}$ es el índice de refracción del material.

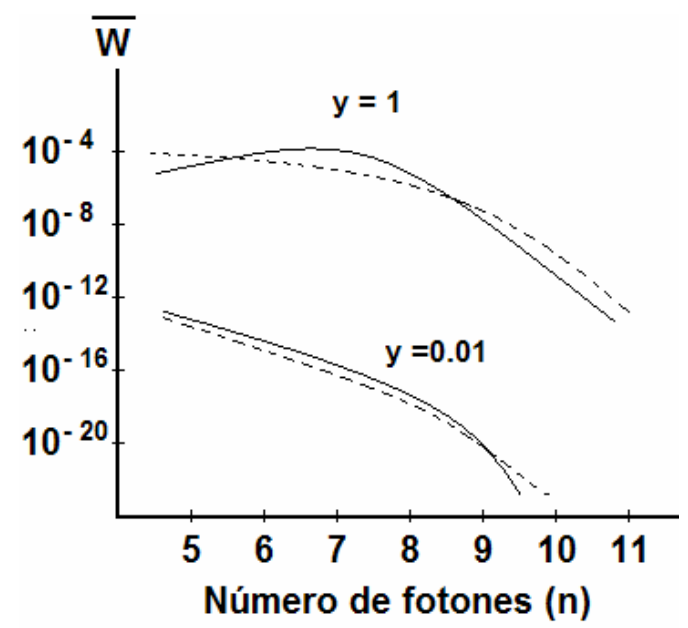

Fig.1. Razones de transición normalizadas en función del número de fotones. Las curvas continuas corresponden a la Ec.(16) y las curvas a trazos a la Ec.(27). El parámetro de intensidad se define por $\mathrm{y}=\mathrm{I} /\left(10^{12} \mathrm{~W} / \mathrm{cm}^{2}\right)$; así que las curvas con $\mathrm{y}=1$ corresponden a $\mathrm{I}=10^{12} \mathrm{~W} / \mathrm{cm}^{2}$ y las curvas con $\mathrm{y}=0,01$ corresponden $\mathrm{I}=10^{10} \mathrm{~W} / \mathrm{cm}^{2}$. 


\section{Discusión de resultados}

Se puede demostrar que para intensidades de láser moderadamente altas $\left(10^{10} \mathrm{~W} / \mathrm{cm}^{2} \leq \mathrm{I} \leq\right.$ $10^{13} \mathrm{~W} / \mathrm{cm}^{2}$ ) la razón de transición obtenida en la aproximación de Volkov, Ec.(16), conduce a los mismos resultados que la razón de transición obtenida por el método convencional de perturbaciones, Ec.(27), para cualquier valor de n.

En la Fig.1 se muestra la gráfica de la razón de transición normalizada $(\bar{w})$ en función del número de fotones (n) de una luz láser de 1050 $\mathrm{nm}\left(\hbar \omega_{\mathrm{L}}=1,19 \mathrm{eV}\right)$ para una muestra de $\mathrm{ZnS}$ que absorbiera rayos-X de 1,1 keV. En la Fig.1 comparando los resultados para las razones de transición $\mathrm{W}^{(\mathrm{n})}$ (curva continua) y $\mathrm{W}_{\mathrm{o}}^{(\mathrm{n})}$ (curva a trazos) para dos intensidades de láser $\left(10^{10} \mathrm{y}\right.$ $10^{12} \mathrm{~W} / \mathrm{cm}^{2}$ ) en función del número de fotones (n) de láser absorbidos, se comprueba aproximadamente la concordancia predichas por las ecuaciones Ec.(16) y (27) para los valores $\mathrm{y}=0,01$ e $\mathrm{y}=1$.

Explorando el comportamiento de las razones de transición normalizadas en la Fig.1, se deduce que la absorción de rayos-X en el $\mathrm{ZnS}$ será un proceso más probable conforme se incrementa la intensidad del láser de acuerdo como lo predicen las Ecs. (16) y (27).

\section{Conclusiones}

Las razones de transición entre bandas de energía fueron calculadas en forma analítica utilizando la aproximación de Volkov y la aproximación dipolar eléctrica para los rayos-X y un láser con polarización circular y de intensidad arbitraria.
Para intensidades de luz láser moderadamente altas, con polarización circular, los resultados para la razón de transición en la aproximación de Volkov son compatibles con los resultados obtenidos por el método convencional de perturbaciones para un sólido de $\mathrm{ZnS}$.

Para otras intensidades de luz láser mucho mayores $(y>>1)$ se espera que las probabilidades de transición tengan un valor máximo para un número específico de fotones lo cual significará una mayor absorción de fotones de luz láser en el metal.

Se ha demostrado en este trabajo que la aproximación de Volkov es útil para investigar teóricamente la fotoionización en metales con rayos-X con absorción multifotónica de un láser intenso.

\section{Referencias}

[1] H. R. Reiss, Phys. Rev. A22, 1786, 1980.

[2] O. Monroy C., Fotoionización de metales. Revista de Investigación de Física. Vol.3, Nº y 2,2000 .

[3] Y. L. Luke, Integrals de Bessel Functions. McGraw Hill, New York, 1962.

[4] F. Ehlotzky, Can. J. Phys. 63, 907, 1985.

[5] M. S. Pindzola, D.C. Griffin and C. Bottcher, Validity of TDHF Theory for the Multiphoton Ionization of Atoms Phys. Rev. Lett. 66, 2305, 1991. 